
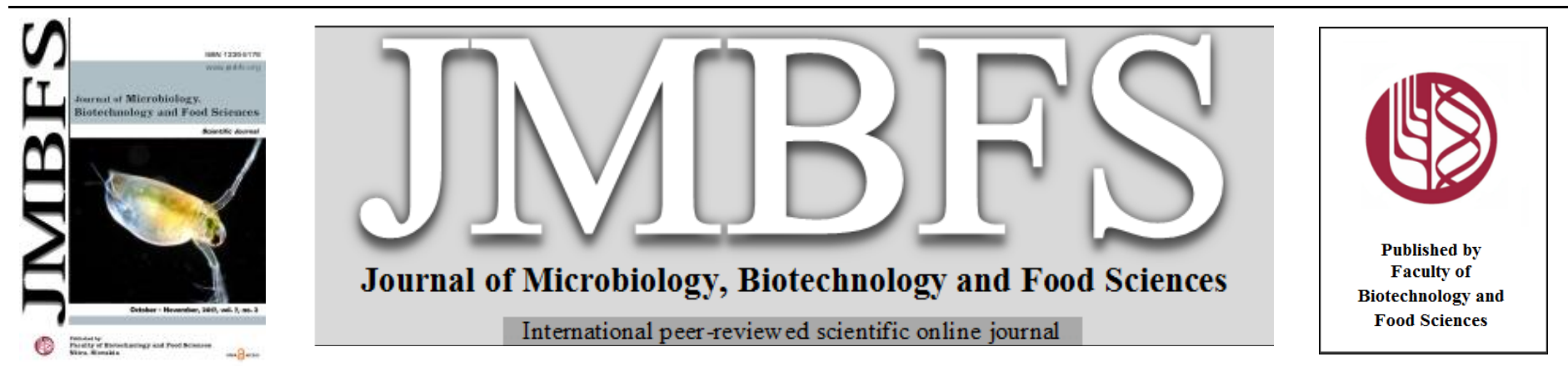

\title{
EXPLORATION OF A HOT SPRING FOR THERMOSTABLE PROTEASE PRODUCERS
}

\section{Anupama Prabhakarrao Pathak*, Mukundraj Govindrao Rathod}

Address(es): Prof. Dr. (Mrs.) Anupama Prabhakarrao Pathak,

School of Life Sciences (DST-FIST \& UGC-SAP Sponsored), Swami Ramanamd Teerth Marathwada University,Nanded-431606,Maharashtra State, +919404732162.

*Corresponding author: anupama.micro@ rediffmail.com

doi: 10.15414/jmbfs.2017.7.2.101-109

\section{ARTICLE INFO}

Received 18. 7. 2016

Revised 22. 7. 2017

Accepted 3. 8. 2017

Published 1. 10. 2017

Regular article

open 2 access

\begin{abstract}
Unkeshwar hot spring of Maharashtra was explored for the isolation of industrially important efficient thermostable protease producers. These isolates were identified based on their morphological characters, microscopic features, enzymatic profile and biochemical characteristics as Bacillus sphaericus APP21, Staphylococcus auricularis APP32, Microbacterium sp. APP41, and Kurthia sp. APP42 from Unkeshwar hot spring. Thermostable protease production was carried out using casein-yeast extract medium at $50{ }^{\circ} \mathrm{C}$. Maximum specific activity of partially purified thermostable protease was recorded from Bacillus sphaericus APP21 (447.75 U/mg). Molecular weight of partially purified proteases from these isolates has been determined in the range of 14-29 KDa. These proteases have showed excellent thermostability in the range of temperature 45 to $65^{\circ} \mathrm{C}$. These proteases were catalytically active in the range of pH 7 to 9 and belonged to the class serine protease. $\mathrm{Km}$ and Vmax values were found in the range $5.26-8.34 \mathrm{mg} / \mathrm{mL}$ and 446-1380 U/mg respectively. The divalent cations $\mathrm{Mg}^{2+}$ and $\mathrm{Mn}^{2+}$ enhanced the catalytic efficiency of these proteases. These proteases showed excellent catalytic efficiency in presence of selected solvents, surfactants and detergents. These properties make them excellent candidates to use in detergent formulation, food processing, and in many biotechnologically important industries where proteolysis is needed at an elevated range of temperature.
\end{abstract}

Keywords: Thermostable protease; Thermostability; Unkeshwar hot spring; SDS-PAGE; Km; Vmax

\section{INTRODUCTION}

A hot spring is produced by the emergence of geothermal heated groundwater from the Earth's crust. Some popular hot springs are Yellowstone national park, (U.S.A.), Suryakund (Bihar, India), Sohna hot spring (Sohna, Delhi), Atri hot spring (Khordha, Odisha), Manikaran hot spring (Himachal Pradesh, India), Cimanggu hot spring (West Java, Indonesia), hypersaline and heliothermal Ekho Lake (East Antarctica),Garampani hot spring (Assam), Unapdev and Sunapdev hot spring (Maharashtra, India), and Bakreswar hot spring (West Bengal, India) (Ulfah et al., 2011; Labrenz et al., 1998).

Proteases from thermophilic sources have been reported to have optimum catalytic activities at the high temperatures. Therefore thermostable proteases are receiving considerable attention for their usefulness in various enzymatic industrial processes. Proteases have found applications in laundry detergents as additives, leather finishing, silk industry, feeds modification, food processing, brewing, pharmaceuticals, diagnostic reagents, peptide synthesis, and silver recovery from $\mathrm{X}$ ray/photographic film. Some thermostable protease producers have been reported from the genera Pyrococcus, Thermococcus, Staphylothermus, Desulfurococcus, Pyrobaculum and Sulfolobus. In archaea, the hyperthermophilic Desulfurococcus strain was reported to produce the extremely thermostable serine protease (Hanzawa et al., 1996). Thermophilic fungi from the genera Achaetomium, Chaetomium, Penicillium, Rhizopus, Sporotrichum, Torula and Rhizomucor have also been reported to produce novel thermostable proteases (Emi et al., 1976). Among bacteria, the genus Bacillus is the dominant source of thermostable proteases.

Here we have selected the terrestrial Unkeshwar hot spring $\left(19^{\circ} 51^{\prime} 21.3^{\prime \prime} \mathrm{N}\right.$ and $\left.78^{\circ} 15^{\prime} 00.9^{\prime \prime} \mathrm{E}\right)$ from Nanded district of Maharashtra state of, India and explored it for the isolation of various thermophiles. Further we have identified the industrially important thermostable protease producers. Production and characterization of thermostable proteases from the selected isolates were also assessed.

\section{MATERIALS AND METHODS}

\section{Isolation and identification of thermophiles}

Water samples from Unkeshwar hot spring were collected in March, August and December 2011 and March 2012. Temperature and $\mathrm{pH}$ of the water samples were recorded individually at the time of collection of samples (Pathak et al., 2014; Jadhav and Pathak, 2015). Abiotic characterization of Unkeshwar hot spring water samples was performed and published (Pathak and Rathod, 2014). Composite water samples were spread on nutrient agar, tryptone-yeast extract agar, tryptone-yeast glucose agar, Vogel Johnson Agar, glucose sodium azide glycerol agar, thiosulphate agar, J agar, brain heart infusion agar, Gram negative agar and Bacillus agar plates and these plates were incubated at $50{ }^{\circ} \mathrm{C}$ to isolate different thermophiles (Pathak and Rathod, 2015).

Typical morphological characters viz. colony shape, size, margin, elevation, consistency, opacity and pigmentation of selected isolates were recorded. Microscopic characters viz. cell shape, cell size, sporulation and motility of selected isolates were observed (Pathak and Rathod, 2015). Optimization of physical parameters for maximum growth of selected isolates was thoroughly assessed (Polkade $\boldsymbol{e t}$ al., 2015). Sugar utilization pattern of selected isolates was determined by using dextrose, fructose, lactose, sucrose, mannitol, maltose, xylose, arabinose, galactose, glycerol, cellobiose, sorbitol, mellibiose, mannose, trehalose, ribose, salicin, rhamnose, inulin, adonitol, and raffinose (Sneath et al., 1986). Catalase and oxidase tests were performed by using $3 \%$ hydrogen peroxide and $1 \%$ tetramethyl-p-phenylenediamine dihydrochloride respectively (Polkade et al., 2015). Amylase, cellulase, gelatinase, pectinase and lipase production ability of selected isolates was evaluated by using starch, cellulose, gelatine, pectin and tributyrin substrates respectively. Antibiotic susceptibility pattern of selected isolates was evaluated using different types of antibiotic discs (HiMedia, Mumbai). The isolates were identified by comparing their morphological, microscopic and physiological characters, sugar utilization and antibiotic sensitivity pattern, and enzyme profile with the standard reference strains from the Bergey's manual of systematic bacteriology (Joshi et al., 2008; Sharma et al., 2009; Pathak and Sardar, 2012; Pathak and Sardar, 2014; Sardar and Pathak, 2014; Jadhav and Pathak, 2015a; Pathak et al., 2015; Pathak et al., 2015a; Hingole and Pathak, 2013; Sharma et al., 2015; 
Khairnar et al., 2012; Kolekar et al., 2013; Dutta et al., 2015; Pathak and Rathod, 2016).

\section{Screening for thermostable protease production}

Morphological distinct selected isolates were inoculated on casein agar plates and incubated at $50{ }^{\circ} \mathrm{C}$ for $24 \mathrm{~h}$. After incubation, acidic $\mathrm{HgCl}_{2}$ solution was flooded on the same plates to examine the protease production efficiency of the isolates Protease production efficiency was calculated by taking ratio of size of zone to colony size. The thermostable protease producers were selected for further studies (Pathak and Rathod, 2015).

\section{Thermostable protease production}

Protease production medium was composed of casein $1.0 \%$, yeast extract $0.3 \%$, peptone $0.5 \%, \mathrm{KH}_{2} \mathrm{PO}_{4} 0.2 \%, \mathrm{NaCl} 0.3 \%, \mathrm{MgSO}_{4} .7 \mathrm{H}_{2} \mathrm{O} 0.005 \%$ and distributed in different conical flasks. $5 \%$ inoculum $\left(11 \times 10^{5} \mathrm{cfu} / \mathrm{mL}\right)$ of each thermostable protease producer was inoculated individually in the flasks. All the flasks were incubated in orbital shaking incubator (CIS-BL 24, Remi Make, Mumbai) at $50{ }^{\circ} \mathrm{C}$ and $120 \mathrm{rpm}$ agitation speed for $72 \mathrm{~h}$ to occur protease production (Gupta and Khare, 2006; Liu et al., 2010).

\section{Extraction and partial purification of thermostable protease}

After $72 \mathrm{~h}$ incubation, the culture broth from each flask was centrifuged at 10,000 $\mathrm{rpm}$ for $10 \mathrm{~min}$ at $4{ }^{\circ} \mathrm{C}$ in cooling centrifuge machine (Remi make, Vasai) Supernatants were collected individually and used as crude protease extracts. Solid ammonium sulphate was added slowly to the each crude protease extract at the rate of $60 \%$ saturation and kept for overnight at $4{ }^{\circ} \mathrm{C}$. The precipitates were collected by centrifuging at $10,000 \mathrm{rpm}$ for $10 \mathrm{~min}$ and dissolved individually in $0.2 \mathrm{M}$ phosphate buffer of $\mathrm{pH}$ 7.0. The dissolved form of precipitate was dialyzed against the same buffer at $4{ }^{\circ} \mathrm{C}$ for $24 \mathrm{~h}$ (Pathak et al., 2014a; Rathod and Pathak, 2014; Rathod and Pathak, 2016a; Osho et al., 2015; Pathak and Rathod, 2013)

\section{Protease assay}

Partially purified protease $(1 \mathrm{ml})$ from each selected isolate was added individually to the test tubes containing $1 \mathrm{ml}$ of $1 \%$ casein solution prepared in $0.2 \mathrm{M}$ phosphate buffer $\mathrm{pH} 7.0$. These mixtures were then incubated at $50{ }^{\circ} \mathrm{C}$ for $10 \mathrm{~min} .3 \mathrm{~mL}$ of $10 \%$ trichloroacetic acid was added in each tube to terminate the reaction. The reaction in blank tube was stopped at zero hours. The supernatants were collected by centrifuging reaction mixtures at $9000 \mathrm{rpm}$ for 10 min. The absorbance of each supernatant was measured as $280 \mathrm{~nm}$. One unit activity of protease was defined as the amount of protease required to liberate 1

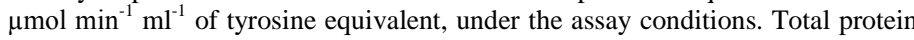
content of each crude protease solution was determined by using BSA as standard (Rathod and Pathak, 2014; Rathod \& Pathak, 2016; Rathod and Pathak, 2017; Osho et al., 2015; Pathak and Rathod, 2013; Pathak et al., 2014b; Lowry et al., 1951).

\section{Molecular weight determination}

Molecular weight of partially purified proteases from each selected isolate was determined by the sodium dodecyl sulphate (SDS) polyacrylamide gel electrophoresis (PAGE; 12\%) as described by Laemmli (1970).Protein broad range molecular weight markers $\left(\mathrm{GeNei}^{\mathrm{TM}}\right.$ Bioscience catalogue no. PMWB 623110475001730) were used to determine molecular weight of partially purified proteases from selected isolates (Rathod and Pathak, 2014a).

\section{Effect of temperature and $\mathrm{pH}$ on catalytic efficiency of proteases}

Effect of temperature on catalytic efficiency of partially purified proteases from selected isolates was determined at the temperature range from 35 to $85{ }^{\circ} \mathrm{C}$. Catalytic efficiency of partially purified proteases from selected isolates was determined at pre-determined optimized temperatures and different $\mathrm{pH}$ values using appropriate buffers of $\mathrm{pH} 4$ to 10 (Rathod and Pathak, 2014; Osho et al., 2015; Pathak and Rathod, 2013; Rathod and Pathak, 2014a).

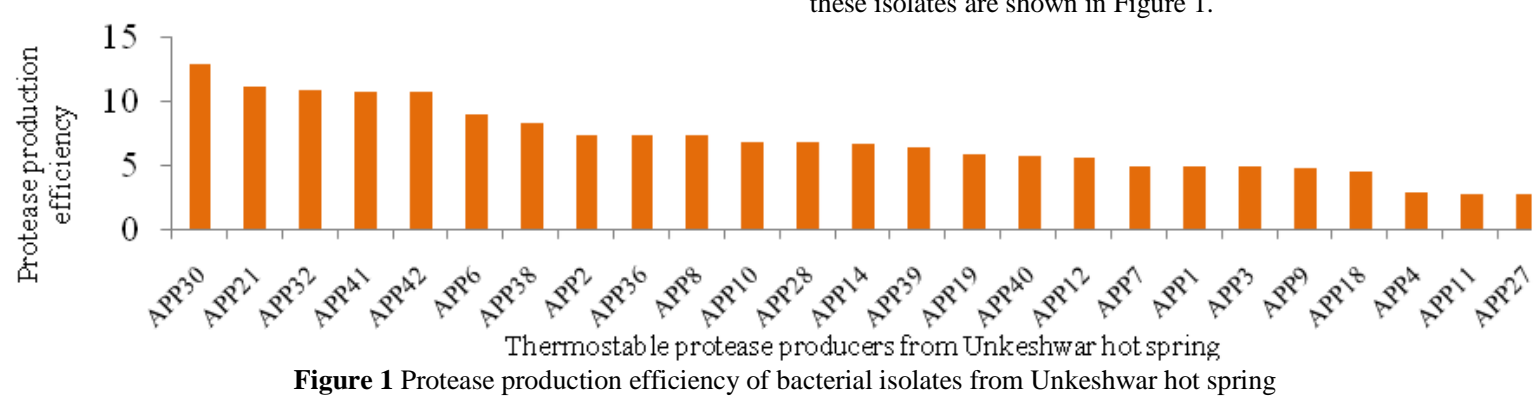

Stability studies of proteases at pre-determined optimum temperature and pH values

Stability of partially purified proteases from selected isolates was examined by incubating the proteases at pre-determined optimum $\mathrm{pH}$ and temperature values for 0-6 hours without substrate and then measuring the residual (remaining) enzyme activity under standard assay conditions. The protease activity before pre-incubation at selected parameters was considered as $100 \%$ and residual (remaining) activity was calculated (Pathak et al., 2014a; Rathod and Pathak, 2014; Pathak and Rathod, 2013; Rathod and Pathak, 2014a).

Effect of different casein concentration on protease activity and determination of $\mathrm{Km}$ and Vmax values

Partially purified proteases from the selected isolates were incubated with buffered casein solutions of different concentrations of casein ranging from 5 to $30 \mathrm{mg} / \mathrm{mL}$. Protease assay was carried out at pre-determined optimum temperature and $\mathrm{pH}$ values of partially purified proteases for $10 \mathrm{~min}$ and protease activity per unit time was determined with each selected substrate concentration. The values of $\mathrm{Km}$ and Vmax of partially purified proteases from the selected isolates were determined after constructing Lineweaver-Burk plots (Rathod and Pathak, 2014).

Effect of selected metal ions on catalytic efficiency of partially purified protease

Effect of inducers and inhibitors on catalytic efficiency of partially purified proteases was determined using $1 \%$ metal solution viz. $\mathrm{FeCl}_{3}, \mathrm{MgCl}_{2}, \mathrm{CuCl}_{2}$ $\mathrm{CaCl}_{2}, \mathrm{ZnCl}_{2}, \mathrm{BaCl}_{2}, \mathrm{HgCl}_{2}, \mathrm{AgNO}_{3}, \mathrm{KCl}, \mathrm{NaCl}$ and $\mathrm{MnCl}_{2}$. Protease activity in absence of metal ions was considered as $100 \%$ and residual protease activities (\%) were calculated (Rathod and Pathak, 2014).

Effect of activators and inhibitors on catalytic efficiency of partially purified protease

Effect of selected solvents (1\%) viz. Chloroform, Ethanol, Butanol, Hexane, Heptane, Tolune, Triton X-100, Acetonitrile, Benzene, Glycerol and Isopropano and other additives viz. Disodium ethylene diamino tetra acetic acid (1\%), Hydrogen peroxide (1\%), Sodium dodecyl sulphate (1\%), 2-Mercaptoethanol (5 $\mathrm{mM})$, Phenyl methyl sulphonyl fluoride $(5 \mathrm{mM})$, Tween $20(1 \%)$ and Tween 80 $(1 \%)$ was also tested on catalytic efficiency of partially purified proteases from the selected isolates. Protease activity in absence of any activators and inhibitors was considered as $100 \%$ and residual protease activities (\%) were calculated (Rathod and Pathak, 2014).

\section{Compatibility of partially purified proteases with detergents}

Catalytic efficiency of partially purified proteases from the selected isolates with commercial detergents was assessed using Ariel ${ }^{\circledR}$ (Procter and Gamble, Suisse), Tide ${ }^{\circledR}$ (Procter and Gamble, Suisse), Rin ${ }^{\circledR}$ (Hindustan Lever Ltd India), Wheel ${ }^{\circledR}$ (Hindustan Lever Ltd India), Surf excel® (Hindustan Unilever Ltd India), Nirma ${ }^{\circledR}$ (Nirma Lever Ltd India), Ghadi ${ }^{\circledR}$ (Kanpur trading Co Pvt Ltd Kanpur, India), Sasa ${ }^{\circledR}$ (Sasa Detergent, Pune) and Vim ${ }^{\circledR}$ (Vim Co Ltd India) at final concentration $7 \mathrm{mg} / \mathrm{mL}$ in partially purified protease solution. The prepared detergent solutions were boiled for $30 \mathrm{~min}$ and cooled prior to use (Rathod and Pathak, 2014).

\section{RESULTS AND DISCUSSION}

\section{Isolation, screening and identification of thermostable protease producers}

The average values of temperature and $\mathrm{pH}$ of Unkeshwar hot spring water samples were recorded as $49.48 \pm 7.07{ }^{\circ} \mathrm{C}$ and $7.92 \pm 0.06$ respectively. Abiotic characterization of Unkeshwar hot spring water samples was performed and published (Pathak and Rathod, 2014). Total 42 thermophiles were isolated from Unkeshwar hot spring water samples and maintained on nutrient agar slants. These isolates were designated as APP1 to APP42. Amongst these, 25 isolates have showed thermostable protease production. Protease production efficiency of these isolates are shown in Figure 1.
Figure 1 Protease production efficiency of bacterial isolates from Unkeshwar hot spring 
Maximum thermostable protease production efficiency was showed by the isolate APP30 followed by the isolates APP21, APP32, APP41, APP42, APP6, APP38, APP2, APP36, APP8, APP10, APP28, APP14, APP39, APP19, APP40, APP12, APP7, APP1, APP3, APP9, APP18, APP4, APP11 and APP27.

Morphological and microscopic characters of APP21, APP32, APP41 and APP42 isolates have been given in Table 1. Sugar-utilization pattern and enzyme profile of these isolates have been given in Table 2 and Table 3 respectively. Optimum conditions required for growth and antibiotic susceptibility pattern of these thermostable protease producers have been given in Table 4 and Table 5 respectively. Antibiotic sensitivity pattern of isolates was used previously by many researchers as one of the taxonomic tools for microbial identification (Lindberg et al., 1977; Sneath et al., 1986).

Table 1 Morphological and microscopic characters of thermostable protease producers from Unkeshwar hot water spring, Maharashtra

\begin{tabular}{|c|c|c|c|c|}
\hline Characters & Bacillus sphaericus APP21 & Staphylococcus auricularis APP32 & Microbacterium sp. APP41 & Kurthia sp. APP42 \\
\hline Shape & Round & Round & Round & Circular \\
\hline Size (mm) & 1 & 1.5 & 2 & 2 \\
\hline Margin & Irregular & Entire & Cerate & Entire \\
\hline Colour & Off white & white & Pale White & Creamy White \\
\hline Elevation & Raised & Raised & Flat & Raised \\
\hline Consistency & Sticky & Sticky & Sticky & Sticky \\
\hline Opacity & Opaque & Opaque & Opaque & Opaque \\
\hline Gram stain & Gram positive & Gram positive & Gram variable & Gram variable \\
\hline \multirow{3}{*}{$\begin{array}{l}\text { Cell Size } \\
(\mu \mathrm{m}) \\
\text { Cell Shape }\end{array}$} & $\mathrm{L}=1.0$ & $\mathrm{D}=1.0$ & $\mathrm{~L}=1.0$ & $\mathrm{~L}=1.0$ \\
\hline & $\mathrm{B}=0.5$ & & $\mathrm{~B}=0.5$ & $\mathrm{~B}=0.5$ \\
\hline & $\begin{array}{l}\text { Coco- } \\
\text { bacillary }\end{array}$ & Coccoid & Rod & Rod \\
\hline Motility & Non motile & Non motile & Motile & Motile \\
\hline Sporulation & + & - & - & - \\
\hline Position of spore & Terminal & - & - & - \\
\hline
\end{tabular}

Table 2 Sugar utilization pattern of thermostable protease producers from Unkeshwar hot water spring, Maharashtra

\begin{tabular}{|c|c|c|c|c|}
\hline $\begin{array}{l}\text { Isolates } \rightarrow \\
\text { Tests } \downarrow\end{array}$ & $\begin{array}{c}\text { Bacillus sphaericus } \\
\text { APP21 }\end{array}$ & $\begin{array}{c}\text { Staphylococcus } \\
\text { auricularis APP32 }\end{array}$ & Microbacterium sp. APP41 & Kurthia sp. APP42 \\
\hline Dextrose & - & $\mathrm{w}+$ & ++ & $\mathrm{w}+$ \\
\hline Fructose & + & - & + & - \\
\hline Lactose & - & - & - & - \\
\hline Sucrose & - & - & + & - \\
\hline Mannitol & - & - & - & - \\
\hline Maltose & - & - & - & ++ \\
\hline Xylose & - & - & + & - \\
\hline Arabinose & - & - & - & - \\
\hline Galactose & - & - & - & - \\
\hline Glycerol & - & - & - & - \\
\hline Cellobiose & - & - & - & - \\
\hline Sorbitol & - & - & - & - \\
\hline Mellibiose & - & - & - & - \\
\hline Mannose & - & + & - & - \\
\hline Trehalose & - & - & + & - \\
\hline Ribose & - & - & + & + \\
\hline Salicin & - & - & + & - \\
\hline Rhamnose & - & - & - & - \\
\hline Adonitol & - & + & - & - \\
\hline Raffinose & - & - & - & - \\
\hline Gas Production & - & - & - & - \\
\hline Indole Test & - & - & - & - \\
\hline MR Test & - & - & - & - \\
\hline VP Test & - & - & - & - \\
\hline Citrate Test & - & - & + & - \\
\hline
\end{tabular}

Table 3 Evaluation of enzyme profile of thermostable protease producers from Unkeshwar hot water spring, Maharashtra

\begin{tabular}{lcccc}
\hline Enzymes & $\begin{array}{c}\text { Bacillus sphaericus } \\
\text { APP21 }\end{array}$ & $\begin{array}{c}\text { Staphylococcus } \\
\text { auricularis APP32 }\end{array}$ & Microbacterium sp. APP41 & Kurthia $\mathrm{sp.} \mathrm{APP42}$ \\
\hline Catalase & + & + & + & + \\
Oxidase & + & ++ & + & + \\
Caseinase & + & + & + & + \\
Amylase & - & - & - & + \\
Gelatinase & + & - & + \\
Cellulase & - & - & - \\
Urease & + & - & - \\
Lipase & - & & - \\
Pectinase & - & & - \\
\hline
\end{tabular}


Table 4 Optimum conditions required for growth of thermostable protease producers from Unkeshwar hot water spring, Maharashtra

\begin{tabular}{lllll}
\hline Parameters & $\begin{array}{l}\text { Bacillus sphaericus } \\
\text { APP21 }\end{array}$ & $\begin{array}{l}\text { Staphylococcus } \\
\text { auricularis APP32 }\end{array}$ & $\begin{array}{l}\text { Microbacterium } \mathrm{sp} . \\
\text { APP41 }\end{array}$ & Kurthia sp. APP42 \\
\hline Temperature growth range $\left({ }^{\circ} \mathrm{C}\right)$ & $25-65$ & $25-45$ & $25-55$ & $30-50$ \\
Optimum temperature $\left({ }^{\circ} \mathrm{C}\right)$ & 55 & 45 & 45 & 50 \\
pH growth range & $7-9$ & $5-8$ & $6-9$ & $6-10$ \\
Optimum pH & 8 & 7 & 8 & 7 \\
Optimum growth period $(\mathrm{h})$ & 24 & 24 & 24 & 72 \\
\hline
\end{tabular}

Table 5 Antibiotic susceptibility pattern of thermostable protease producers from Unkeshwar hot water spring

\begin{tabular}{lcccc}
\hline $\begin{array}{l}\text { Isolates } \rightarrow \\
\text { Tests } \downarrow\end{array}$ & $\begin{array}{c}\text { Bacillus sphaericus } \\
\text { APP21 }\end{array}$ & $\begin{array}{c}\text { Staphylococcus } \\
\text { auricularis APP32 }\end{array}$ & $\begin{array}{c}\text { Microbacterium } \text { sp. } \\
\text { APP41 }\end{array}$ & $\begin{array}{c}\text { Kurthia sp. } \\
\text { APP42 }\end{array}$ \\
\hline Amikacin $(30 \mu \mathrm{g})$ & 2.5 & $\mathrm{R}$ & 3.5 & 3.3 \\
Ampicillin $(10 \mu \mathrm{g})$ & 4.0 & 3.5 & 3.5 & 2.4 \\
Cefoperazone $(75 \mu \mathrm{g})$ & 2.6 & $\mathrm{R}$ & 2.5 & 2.1 \\
Cefoxitin $(30 \mu \mathrm{g})$ & 1.0 & 2.5 & 2.5 & 2.5 \\
Ceftazidime $(30 \mu \mathrm{g})$ & 2.5 & 3.5 & 2.1 \\
Ceftriaxon $(30 \mu \mathrm{g})$ & 2.2 & $\mathrm{R}$ & 4.0 & 2.3 \\
Chloramphenicol $(25 \mu \mathrm{g})$ & 2.4 & 2.7 & 3.6 & 2.6 \\
Chloramphenicol $(30 \mu \mathrm{g})$ & 2.2 & 2.2 & 3.3 & 2.6 \\
Ciprofloxacin $(5 \mu \mathrm{g})$ & 2.1 & 3.4 & 3.2 & 2.7 \\
Cotrimoxazole $(30 \mu \mathrm{g})$ & 2.4 & 2.6 & 3.5 & 1.9 \\
Gentamicin $(10 \mu \mathrm{g})$ & 2.5 & 3.0 & 3.1 & 3.0 \\
Netillin $(30 \mu \mathrm{g})$ & 2.6 & $\mathrm{R}$ & 4.2 & 2.8 \\
Ofloxacin $(5 \mu \mathrm{g})$ & 2.8 & 1.2 & 2.7 & 1.8 \\
Penicillin $(10 \mathrm{U})$ & 2.1 & 3.5 & $\mathrm{R}$ \\
Penicillin $\mathrm{G}(1 \mathrm{U})$ & 4.2 & $\mathrm{R}$ & 3.0 & 1.9 \\
Piperacillin $(100 \mu \mathrm{g})$ & 2.5 & 1.6 & 2.0 & 2.7 \\
Streptomycin $(10 \mu \mathrm{g})$ & 2.6 & 1.8 & 3.5 & 2.2 \\
Sulphatriad $(30 \mu \mathrm{g})$ & $\mathrm{R}$ & 2.9 & 3.0 & 3.0 \\
Teicoplanin $(30 \mu \mathrm{g})$ & 3.5 & $\mathrm{R}$ & $\mathrm{R}$ & 3.0 \\
Tetracycline $(30 \mu \mathrm{g})$ & 2.4 & 3.7 & 1.9 & 2.4 \\
Tetracycline $(25 \mu \mathrm{g})$ & 2.4 & 2.0 & $\mathrm{R}$ & 2.8 \\
Vancomycin $(30 \mu \mathrm{g})$ & 2.0 & 1.0 & 3.7 & \\
\hline Size & 2.3 & & &
\end{tabular}

Size of zone of inhibition is given in centimeter, $R=$ Resistant.

The isolates APP21, APP32, APP41 and APP42 were identified as Bacillus sphaericus, Staphylococcus auricularis, Microbacterium sp. and Kurthia sp. respectively based on their morphological and microscopic characters, sugar utilization pattern, enzyme profile and antibiotic susceptibility pattern (Table 1 to 5). In our previous studies, the isolates APP1, APP2, APP3 and APP4 were identified as Bacillus alvei, Bacillus brevis, Bacillus sterothermophilus and Bacillus subtilis respectively (Pathak and Rathod, 2014).APP6 was previously identified as Bacillus firmus (Pathak et al., 2014c). The isolates APP7, APP8, APP9, APP10, APP11, APP12 and APP14 were previously identified as Bacillus licheniformis, Bacillus megaterium, Actinobacillus hominis, Lysinibacillus sphaericus, Paenibacillus alvei, Bacillus simplex and Pseudomonas fragii respectively (Pathak and Rathod, 2015). The isolates APP18, APP27 and APP40 were previously identified as Bacillus cereus, Bacillus stearothermophilus and Microbacterium sp. respectively and communicated in a journal. The isolate APP19 was previously identified as Bacillus atrophaeus (Pathak et al., 2014b). The isolates APP28, APP30, APP38 and APP39 were previously identified as Bacillus brevis, Pseudomonas oleovorans, Bacillus aminovorans and Bacillus firmus and published in a journal (Pathak and
Rathod, 2015a). The isolate APP36 was previously identified as Bacillus subtilis (Pathak and Rathod, 2015b).

\section{Thermostable protease production}

The data regarding total activity, total protein content, specific activity, purification fold and yield of partially purified proteases from selected isolates are given in Table 6. Maximum specific activity of partially purified thermostable protease was recorded from Bacillus sphaericus APP21 (447.75 U/mg) followed by Staphylococcus auricularis APP32, Microbacterium sp. APP41, Kurthia sp APP42, Bacillus atrophaeus APP19, Bacillus stearothermophilus APP27, Bacillus brevisAPP28, Bacillus cereus APP18, Bacillus aminovorans APP38, Bacillus simplex APP12, Bacillus subtilis APP36, Paenibacillus alvei APP11, Bacillus subtilis APP4, Bacillus firmus APP6, Bacillus licheniformis APP7, Bacillus brevis APP2, Bacillus sterothermophilus APP3, Bacillus firmus APP39, Actinobacillus hominis APP9, Microbacterium sp. APP40, Pseudomonas fragiiAPP14, Bacillus megaterium APP8, Lysinibacillus sphaericus APP10 and Bacillus alvei APP1.

Table 6 Thermostable protease production, partial purification and yield obtained from selected isolates from Unkeshwar hot spring, Maharashtra

\begin{tabular}{|c|c|c|c|c|c|}
\hline \multicolumn{6}{|c|}{ Partially purified protease } \\
\hline Thermostable protease producers $\downarrow$ & $\begin{array}{l}\text { Total activity } \\
\text { (Units/mL) }\end{array}$ & $\begin{array}{c}\text { Total } \\
\text { protein } \\
(\mathrm{mg} / \mathrm{mL})\end{array}$ & $\begin{array}{l}\text { Specific } \\
\text { activity } \\
\text { (U/mg } \\
\text { protein) }\end{array}$ & $\begin{array}{l}\text { Purification } \\
\quad \text { (fold) }\end{array}$ & $\begin{array}{c}\text { Yield } \\
(\%)\end{array}$ \\
\hline Bacillus sphaericus APP21 & 1298.5 & 2.9 & 447.75 & 2.6 & 76 \\
\hline Staphylococcus auricularis APP32 & 646.33 & 1.45 & 445.74 & 2.9 & 89 \\
\hline Microbacterium sp. APP41 & 875.76 & 2 & 437.88 & 2.2 & 72 \\
\hline Kurthia sp. APP42 & 512.67 & 1.6 & 320.41 & 2.5 & 71 \\
\hline Bacillus firmus APP6 & 756.345 & 7.5 & 100.85 & 2.23 & 28 \\
\hline Bacillus aminovorans APP38 & 923.45 & 6.4 & 144.29 & 2.14 & 52 \\
\hline Bacillus brevis APP2 & 656.75 & 7.3 & 89.97 & 2.5 & 37 \\
\hline Bacillus subtilis APP36 & 523.24 & 4.0 & 130.81 & 2.54 & 62 \\
\hline Bacillus megaterium APP8 & 240.45 & 5.8 & 41.46 & 2.0 & 75 \\
\hline Lysinibacillus sphaericus APP10 & 105.64 & 2.8 & 37.73 & 1.34 & 47 \\
\hline
\end{tabular}


Bacillus brevis APP28

Pseudomonas fragiiAPP14

Bacillus firmus APP39

Bacillus atrophaeus APP19

Microbacterium sp.APP40

Bacillus simplex APP12

Bacillus licheniformis APP7

Bacillus alvei APP1

Actinobacillus hominis APPO

Bacillus cereus APP18

Bacillus subtilis APP4

Paenibacillus alvei APP11

Bacillus stearothermophilus APP27
Bacillus sterothermophilus APP3

$\begin{array}{lcccc}795.89 & 4.7 & 169.34 & 2.63 & 62 \\ 346.65 & 7.4 & 46.84 & 1.76 & 65 \\ 235.65 & 3.5 & 67.33 & 2.76 & 84 \\ 498.67 & 1.6 & 311.66 & 2.8 & 71 \\ 235.87 & 3.8 & 62.07 & 2.75 & 74 \\ 466.87 & 3.5 & 133.39 & 1.08 & 46 \\ 346.75 & 3.8 & 91.25 & 1.09 & 85 \\ 234.00 & 7.4 & 31.62 & 1.56 & 45 \\ 754.63 & 9.4 & 80.28 & 1.45 & 58 \\ 235.76 & 3.7 & 63.72 & 1.76 & 37 \\ 302.56 & 1.9 & 159.24 & 2.65 & 73 \\ 664.53 & 6.2 & 107.18 & 1.67 & 56 \\ 563.20 & 4.5 & 125.16 & 1.65 & 27 \\ 357.8 & 2 & 178.9 & 2 & 68\end{array}$

\section{Molecular weight determination}

Molecular weight of partially purified thermostable proteases from Bacillus sphaericus APP21, Staphylococcus auricularis APP32, Microbacterium sp. APP41, and Kurthia sp. APP42 were determined as approximately $29 \mathrm{KDa}, 14.3$ $\mathrm{KDa}, 19 \mathrm{KDa}$ and 20.1 KDa respectively (Figure 2). Anandharaj et al. (2016) isolated thermostable protease producer Bacillus alkalitelluris TWI3, from tannery waste and reported $42.6 \mathrm{kDa}$ molecular weight of TWI3 protease by SDS-PAGE. Ansari et al. (2015) isolated a thermophilic bacterium Aneurinibacillus thermoaerophilus MCW220 from Manikaran hot spring, Himachal pradesh (India). MCW220 thermostable protease showed optimum pH 9 and temperature $50{ }^{\circ} \mathrm{C}$. Molecular weight of MCW220 protease was $45 \mathrm{kDa}$ that is comparatively greater than the molecular weights of proteases from APP21, APP32, APP41 and APP42. Yildirim et al. (2017) reported $38.55 \mathrm{kDa}$ molecular weight of thermostable protease from Aeribacillus pallidus.

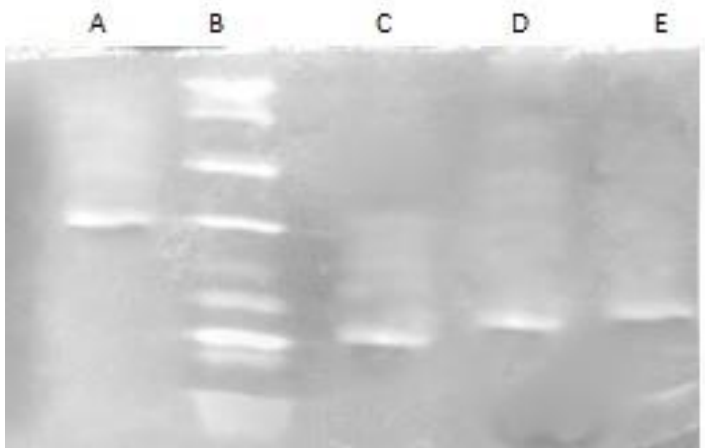

$205 \mathrm{kDa}$

$97.4 \mathrm{KDa}$

$66 \mathrm{Kda}$

$43 \mathrm{KDa}$

$29 \mathrm{KDa}$

$20.1 \mathrm{KDa}$

$14.3 \mathrm{KDa}$

$6.5 \mathrm{KDa}$

$3.5 \mathrm{KDa}$

Figure 2 Determination of molecular weightsof partially purified thermostable proteases from selected isolates from Unkeshwar hot spring as revealed by $12 \%$ SDS-PAGE

Lane A= Partially purified thermostable protease from Bacillus sphaericus APP21 (29 KDa) Lane $\mathrm{B}=$ Protein broad range molecular weight markers $\left(\mathrm{GeNe}^{\mathrm{TM}}\right.$ Bioscience catalogue no. PMWB 623110475001730)

Lane C = Partially purified thermostable protease from Staphylococcus auricularis APP32 $(14.3 \mathrm{KDa})$

Lane D=Partially purified thermostable protease from Microbacterium sp. APP41 (Approx. $19 \mathrm{KDa})$

Lane E=Partially purified thermostable protease from Kurthia sp. APP42 (20.1 KDa)

\section{Effect of temperature and $\mathrm{pH}$ on catalytic efficiency of proteases}

Maximum catalytic efficiency of partially purified proteases from Staphylococcus auricularis APP32 and Kurthia sp. APP42 was found at temperature $55^{\circ} \mathrm{C}$. Maximum catalytic efficiency of partially purified proteases from Bacillus sphaericus APP21 was found at temperature $65{ }^{\circ} \mathrm{C}$. Maximum catalytic efficiency of partially purified proteases from Microbacterium sp. APP41 was found at temperature $45{ }^{\circ} \mathrm{C}$ (Figure 3). Selim (2015) reported thermostable alkaline protease production from Bacillus aryabhattai J4. This protease exhibited optimum temperature $60{ }^{\circ} \mathrm{C}$ and showed stability at a broad $\mathrm{pH}$ range 6 10. Antranikian and Klingeberg (1991) published a patent on thermostable protease from Thermococcus celer. This protease showed optimum catalytic activity at temperature range $75-100{ }^{\circ} \mathrm{C}$. Dammak et al. (2016) reported optimum temperature and $\mathrm{pH}$ of thermostable protease from Halorubrum ezzemulense strain ETR14 as 9 and $60{ }^{\circ} \mathrm{C}$ respectively.

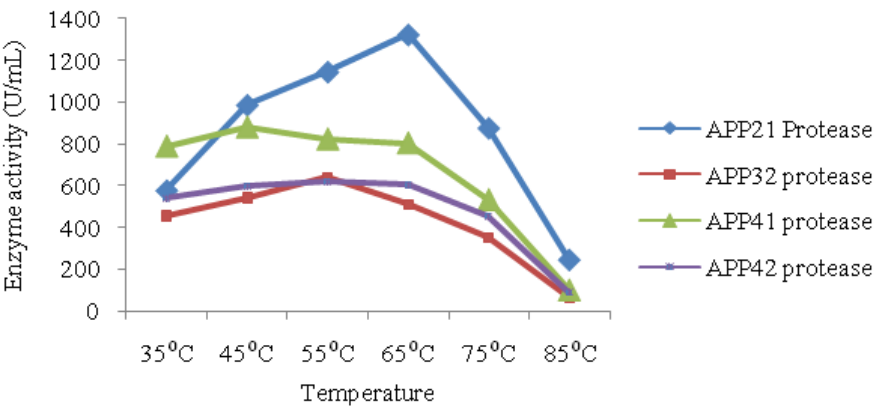

Figure 3 Effect of temperature on catalytic activity of protease

Partially purified proteases from APP21, APP32, APP41 and APP42 have showed remarkable catalytic activity at alkaline $\mathrm{pH}$. Partially purified protease from Staphylococcus auricularis APP32 has showed maximum catalytic efficiency $(1056 \mathrm{U} / \mathrm{mL})$ at $\mathrm{pH}$ 8. Partially purified protease from Bacillus sphaericus APP21 has showed maximum catalytic efficiency $(1543 \mathrm{U} / \mathrm{mL})$ at $\mathrm{pH}$ 9. Maximum catalytic efficiency of partially purified proteases, $880 \mathrm{U} / \mathrm{mL}$ and $620 \mathrm{U} / \mathrm{mL}$, respectively from Microbacterium sp. APP41 and Kurthia sp. APP42 was found at the $\mathrm{pH}$ value 7.0 (Figure 4).

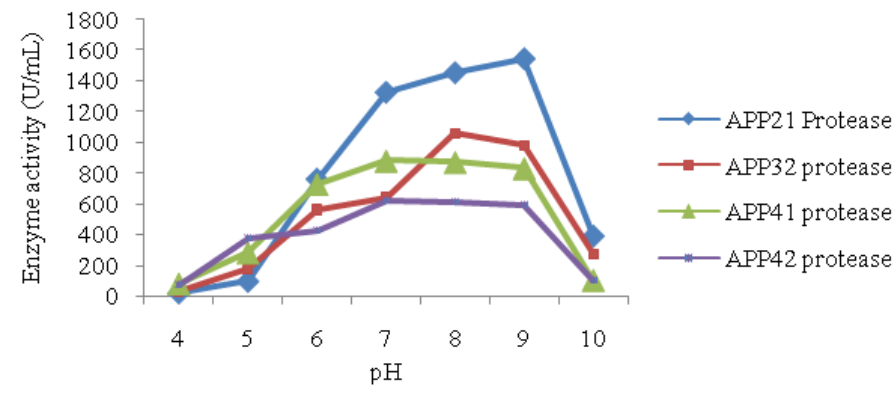

Figure 4 Effect of $\mathrm{pH}$ on catalytic activity of protease

Stability studies of proteases at pre-determined optimum temperature and pH values

Partially purified proteases from Bacillus sphaericus APP21, Staphylococcus auricularis APP32, Microbacterium sp. APP41, and Kurthia sp. APP42 exhibited greater than $50 \%$ of their remaining activity even after $4 \mathrm{~h}$ of incubation period at pre-determined optimum temperature and $\mathrm{pH}$ values (Figure 5). Partially purified protease from Bacillus sphaericus APP21 exhibited 70.84, 63.71, 56.51, $50.55,6.55$ and $4.21 \%$ of residual activity after $1,2,3,4,5$ and $6 \mathrm{~h}$ of preincubation period at $\mathrm{pH} 9.0$ and temperature $65^{\circ} \mathrm{C}$ respectively. Partially purified protease from Staphylococcus auricularis APP32 exhibited 95.83, 92.99, 88.64 $77.75,27.56$ and $8.24 \%$ of residual activity after $1,2,3,4,5$ and 6 h of preincubation period at $\mathrm{pH} 8.0$ and temperature $55^{\circ} \mathrm{C}$ respectively. Partially purified proteases from Microbacterium sp. APP41 exhibited 94.77, 93.18, 91.14, 83.18, 65.23 , and $23.07 \%$ of residual activity after $1,2,3,4,5$ and $6 \mathrm{~h}$ of pre-incubation period at $\mathrm{pH} 7.0$ and temperature $45{ }^{\circ} \mathrm{C}$ respectively. Partially purified proteases from Kurthia sp. APP42 exhibited 98.71, 94.19, 70.48, 63.23, 16.45 and $7.26 \%$ of residual activity after $1,2,3,4,5$ and $6 \mathrm{~h}$ of pre-incubation period at $\mathrm{pH} 7.0$ and temperature $55{ }^{\circ} \mathrm{C}$ respectively (Figure 5). Jordan and Lin (1993) published a patent on thermostable acid protease, thermopsin, from Sulfolobus acidocaldarius. This protease exhibited thermostable activity at $\mathrm{pH}$ range 1-11 and at temperatures between 0 to $100^{\circ} \mathrm{C}$. 


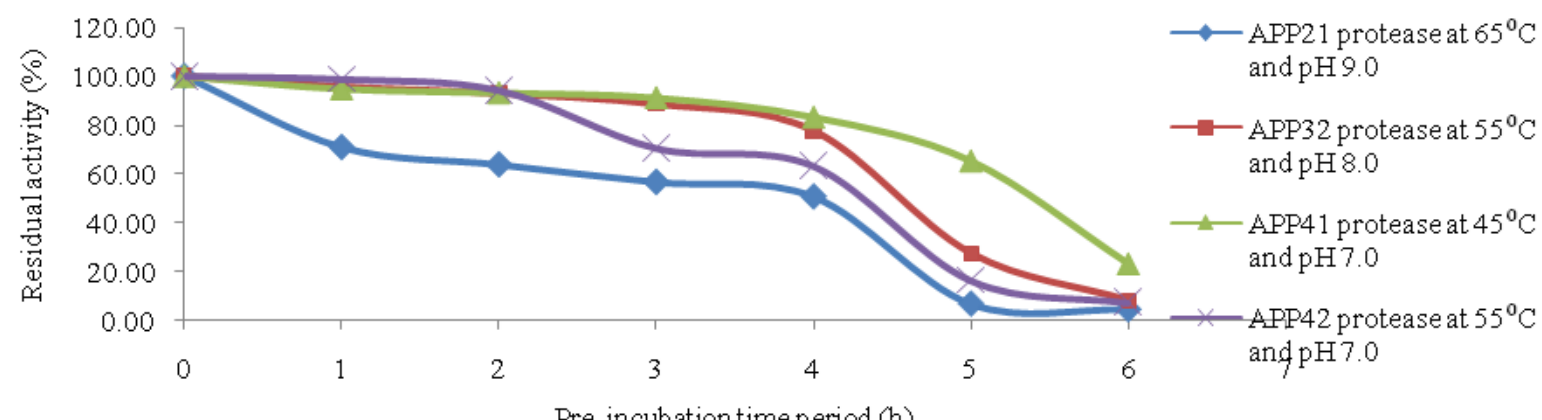

Figure 5 Stability of proteases at pre-determined optimum temperature and $\mathrm{pH}$ values

Effect of different casein concentration on protease activity and determination of $\mathrm{Km}$ and Vmax values

Partially purified proteases from Bacillus sphaericus APP21, Staphylococcus auricularis APP32, Microbacterium sp. APP41 and Kurthia sp. APP42 have showed maximum proteolytic activity at $10,20,15$ and $25 \mathrm{mg} / \mathrm{mL}$ of casein concentrations respectively. The Lineweaver-Burk plots have been reflected in Figure 6 to Figure 9 and $\mathrm{Km}$ and Vmax values of these proteases have been given in Table 7. Thebti et al., 2016 reported $\mathrm{Km}$ and Vmax values of thermostable protease from Geobacillus toebii strain LBT 77 as $1 \mathrm{mg} / \mathrm{mL}$ and $217.5 \mathrm{U} / \mathrm{mL}$ respectively.

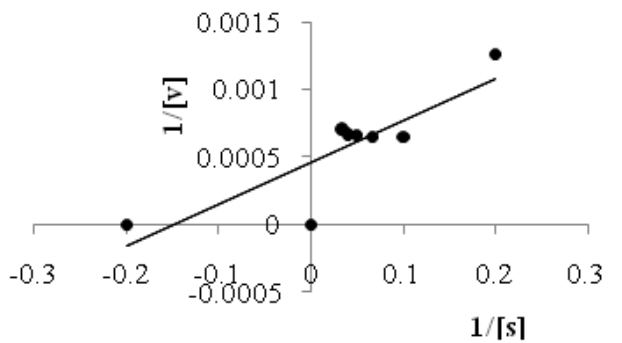

Figure 6 Lineweaver Burk plot for Bacillus sphaericus APP21 protease

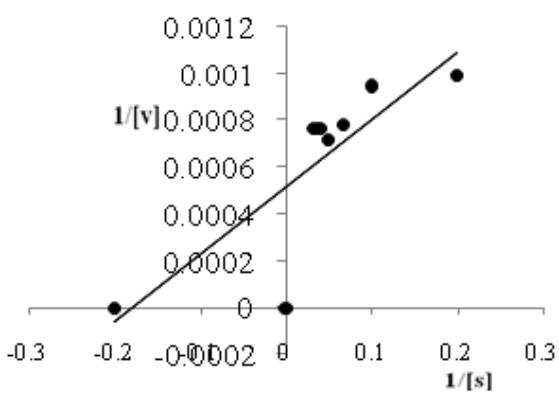

Figure 7 Lineweaver Burk plot for Staphylococcus auricularis APP32 protease

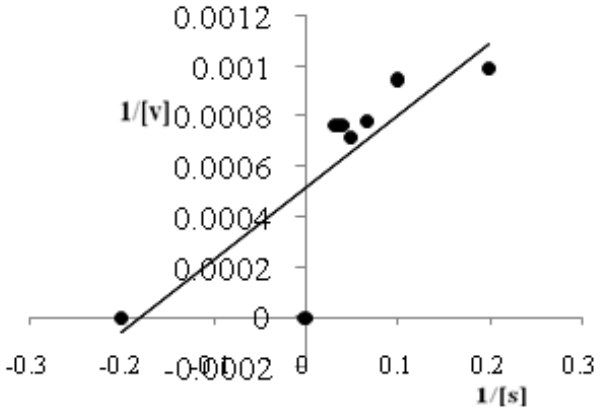

Figure 8 Lineweaver Burk plot for Microbacterium sp. APP41 protease

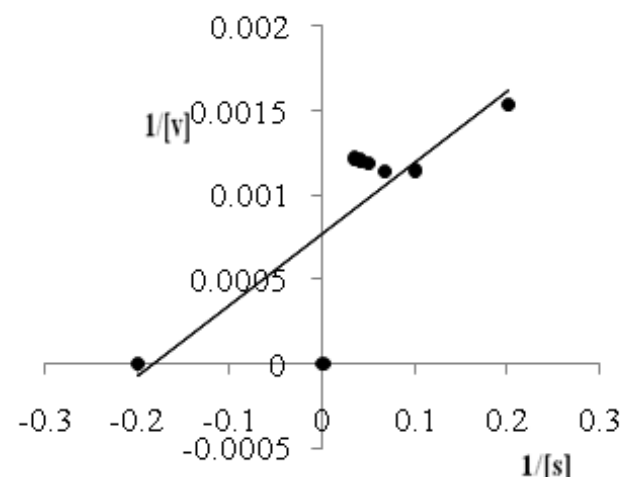

Figure 9 Lineweaver Burk plot for Kurthia sp. APP42 protease

Table 7 Optimum casein concentration for protease activity and $\mathrm{Km}$ and Vmax values of thermostable proteases from microbial isolates from Unkeshwar hot spring

\begin{tabular}{lcc}
\hline $\begin{array}{l}\text { Thermostable proteases from Unkeshwar hot spring } \\
\text { isolates }\end{array}$ & $\begin{array}{l}\text { Optimum casein concentration for } \\
\text { protease activity (mg/mL) }\end{array}$ & $\begin{array}{l}\text { Vmax } \\
\text { (U/mg) }\end{array}$ \\
\hline Bacillus sphaericus APP21 & 10 & $\begin{array}{l}\text { Km } \\
(\mathbf{m g} / \mathbf{m L})\end{array}$ \\
Staphylococcus auricularis APP32 & 20 & 766.28 \\
Microbacterium sp. APP41 & 15 & 1379.31 \\
Kurthia sp. APP42 & 25 & 735.29 \\
\hline
\end{tabular}

Effect of inducers and inhibitors on catalytic efficiency of partially purified protease

Residual activities of partially purified proteases from Bacillus sphaericus APP21, Staphylococcus auricularis APP32, Microbacterium sp. APP41 and Kurthia sp. APP42 in presence of selected metal ions have been given in Table 8 . Catalytic activity of partially purified protease from Bacillus sphaericus APP21was enhanced by $20,5,25$ and $6 \%$ in presence of $\mathrm{Mg}^{2+}, \mathrm{Ca}^{2+}, \mathrm{Ba}^{2+}$ and $\mathrm{Na}^{+}$cations respectively. Catalytic activity of partially purified protease from Staphylococcus auricularis APP32 was enhanced by 30,10,11, 4 and $9 \%$ in presence of $\mathrm{Mg}^{2+}, \mathrm{Ca}^{2+}, \mathrm{Ba}^{2+}, \mathrm{K}^{+}$and $\mathrm{Mn}^{2+}$ cations respectively. Catalytic activity of partially purified protease from Microbacterium sp. APP41 was enhanced by $25,7,4,1$ and $5 \%$ in presence of $\mathrm{Mg}^{2+}, \mathrm{Ca}^{2+}, \mathrm{Ba}^{2+}, \mathrm{Na}^{+}$and $\mathrm{Mn}^{2+}$ cations respectively. Catalytic activity of partially purified protease from Kurthia sp.
APP42 was enhanced by 33 and $7 \%$ in presence of $\mathrm{Mg}^{2+}$ and $\mathrm{Mn}^{2+}$ cations respectively. Thebti et al., 2016 reported that, catalytic activity of thermostable, haloalkaline, solvent stable, and detergent compatible serine protease from Geobacillus toebii Strain LBT 77 was enhanced in presence of $\mathrm{Mg}^{2+}$ and $\mathrm{Ca}^{2+}$ cations. 
Table 8 Residual activities of partially purified proteases in presence of selected metal ions

\begin{tabular}{|c|c|c|c|c|}
\hline $\begin{array}{l}\text { Metal ions } \\
(1 \%) \\
\end{array}$ & $\begin{array}{l}\text { Bacillus sphaericus APP21 } \\
\text { protease }\end{array}$ & $\begin{array}{c}\text { Staphylococcus auricularis } \\
\text { APP32 protease }\end{array}$ & $\begin{array}{l}\text { Microbacterium sp. APP41 } \\
\text { protease }\end{array}$ & $\begin{array}{c}\text { Kurthia } \text { sp. } \\
\text { APP42 protease }\end{array}$ \\
\hline $\mathrm{Mg}^{2+}$ & 120 & 130 & 125 & 133 \\
\hline $\mathrm{Ca}^{2+}$ & 105 & 110 & 107 & 100 \\
\hline $\mathrm{Zn}^{2+}$ & 92 & 98 & 91 & 100 \\
\hline $\mathrm{Hg}^{2+}$ & 80 & 88 & 90 & 92 \\
\hline $\mathrm{Ag}^{3+}$ & 90 & 92 & 94 & 88 \\
\hline $\mathrm{K}^{+}$ & 100 & 104 & 97 & 100 \\
\hline $\mathrm{Na}^{+}$ & 106 & 100 & 101 & 98 \\
\hline $\mathrm{Mn}^{2+}$ & 110 & 109 & 105 & 107 \\
\hline
\end{tabular}

Effect of Effect of inducers and inhibitors on catalytic efficiency of partially purified protease

Catalytic activity of partially purified proteases from Bacillus sphaericus APP21, Staphylococcus auricularis APP32, Microbacterium sp. APP41 and Kurthia sp. APP42 was completely inhibited in presence of $5 \mathrm{mM}$ phenyl methyl sulphonyl fluoride, which indicates that these proteases belong to the class serine proteases. These proteases have showed remarkable catalytic efficiency in presence of selected solvents and surfactants. Residual activities of these partially purified proteases in presence of selected activators and inhibitors have been presented in Table 9.

$\underline{\text { Table } 9 \text { Residual activities of partially purified proteases in presence of selected activators and inhibitors }}$

\begin{tabular}{|c|c|c|c|c|}
\hline Solvents $(1 \%)$ & $\begin{array}{l}\text { Bacillus sphaericus } \\
\text { APP21protease }\end{array}$ & $\begin{array}{l}\text { Staphylococcus } \\
\text { auricularis APP32 } \\
\text { protease }\end{array}$ & $\begin{array}{l}\text { Microbacterium sp. } \\
\text { APP41 protease }\end{array}$ & $\begin{array}{l}\text { Kurthia sp. APP42 } \\
\text { protease }\end{array}$ \\
\hline Chloroform & 68 & 69 & 63 & 73 \\
\hline Ethanol & 96 & 94 & 73 & 75 \\
\hline Butanol & 86 & 84 & 83 & 86 \\
\hline Hexane & 94 & 64 & 96 & 92 \\
\hline Heptane & 86 & 88 & 89 & 93 \\
\hline Tolune & 83 & 86 & 82 & 88 \\
\hline Triton X-100 & 98 & 94 & 93 & 95 \\
\hline Acetonitrile & 75 & 78 & 85 & 75 \\
\hline Benzene & 84 & 86 & 88 & 82 \\
\hline Glycerol & 94 & 92 & 99 & 100 \\
\hline Isopropanol & 91 & 92 & 83 & 84 \\
\hline Na2EDTA $(1 \%)$ & 90 & 94 & 98 & 100 \\
\hline Hydrogen peroxide (1\%) & 88 & 84 & 97 & 89 \\
\hline Sodium dodecyl sulphate $(1 \%)$ & 98 & 99 & 97 & 100 \\
\hline 2-Mercaptoethanol (5 mM) & 87 & 90 & 92 & 91 \\
\hline $\operatorname{PMSF}(5 \mathrm{mM})$ & 0 & 0 & 0 & 0 \\
\hline Tween $20(1 \%)$ & 102 & 104 & 106 & 100 \\
\hline Tween $80(1 \%)$ & 98 & 96 & 97 & 95 \\
\hline Control & 100 & 100 & 100 & 100 \\
\hline
\end{tabular}

\section{Compatibility of partially purified proteases with detergents}

Residual activities of partially purified proteases from Bacillus sphaericus APP21, Staphylococcus auricularis APP32, Microbacterium sp. APP41 and Kurthia sp. APP42 in presence of selected activators and inhibitors have been presented in Table 10. These proteases have showed remarkable catalytic efficiencies in presence of all selected detergents. Partially purified protease from
Bacillus sphaericus APP21 has showed maximum $100 \%$ compatibility with Ariel ${ }^{\circledR}$ and Nirma ${ }^{\circledR}$. Partially purified protease from Staphylococcus auricularis APP32 has showed maximum $98 \%$ compatibility with Vim ${ }^{\circledR}$. Partially purified protease from Microbacterium sp. APP41 and Kurthia sp. APP42 have showed

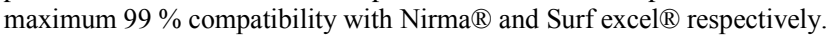

Table 10 Residual activities of partially purified proteases in presence of selected commercial detergents

\begin{tabular}{|c|c|c|c|c|}
\hline $\begin{array}{l}\text { Detergents } \\
(7 \mathrm{mg} / \mathrm{mL})\end{array}$ & $\begin{array}{l}\text { Bacillus sphaericus } \\
\text { APP21 protease }\end{array}$ & $\begin{array}{l}\text { Staphylococcus auricularis } \\
\text { APP32protease }\end{array}$ & $\begin{array}{l}\text { Microbacterium sp. } \\
\text { APP41 protease }\end{array}$ & $\begin{array}{l}\text { Kurthia } \text { sp. } \\
\text { APP42protease }\end{array}$ \\
\hline Ariel $\AA$ & 100 & 97 & 91 & 93 \\
\hline Tide $\mathbb{R}$ & 97 & 93 & 94 & 94 \\
\hline $\operatorname{Rin}{ }^{\circledR}$ & 98 & 91 & 95 & 92 \\
\hline Wheel ${ }^{\circledR}$ & 94 & 80 & 93 & 94 \\
\hline Surf excel $®$ & 99 & 93 & 94 & 99 \\
\hline Nirma ${ }^{\circledR}$ & 100 & 92 & 99 & 93 \\
\hline Ghadi ${ }^{\circledR}$ & 93 & 95 & 92 & 94 \\
\hline $\mathrm{Sasa}{ }^{\circledR}$ & 95 & 92 & 91 & 92 \\
\hline $\operatorname{Vim}{ }^{\circledR}$ & 92 & 98 & 93 & 89 \\
\hline Control & 100 & 100 & 100 & 100 \\
\hline
\end{tabular}




\section{CONCLUSIONS}

Efficient thermostable protease producers were isolated from Unkeshwar ho spring of Maharashtra and identified as Bacillus sphaericus APP21, Staphylococcus auricularis APP32, Microbacterium sp. APP41 and Kurthia sp. APP42. Partially purified proteases from these isolates exhibited excellent thermostability above $45{ }^{\circ} \mathrm{C}$ temperature even after $4 \mathrm{~h}$ of pre-incubation period. These proteases were catalytically active at neutral to alkaline range of $\mathrm{pH}$. These proteases have showed remarkable efficiency in presence of selected metal ions, solvents, surfactants and detergents. These proteases can be used in detergent formulation, food processing, peptide synthesis in presence of solvents and where proteolysis at elevated range of temperature is essential. The thermostable proteases producers from Unkeshwar hot spring can be exploited for production of many other thermostable enzymes beside protease

Acknowledgments: Authors would like to thank University Grants Commission, New Delhi (India) for financial support and Hon. Vice-Chancellor, S.R.T.M. University, Nanded for providing infrastructure and necessary facilities.

\section{REFERENCES}

Anandharaj, M., Sivasankari, B., Siddharthan, N., Rani, R. P., \& Sivakumar, S. (2016). Production, Purification, and Biochemical Characterization of Thermostable Metallo-Protease from Novel Bacillus alkalitelluris TWI3 Isolated from Tannery Waste. Applied biochemistry and biotechnology, 178(8), 1666 1686. http://dx.doi.org/10.1007/s12010-015-1974-7

Ansari, Z., Verma, A., Dhiman, K., Sharma, A., \& Shirkot, P. (2015) Thermostable protease production by Aneurinibacillus thermoaerophilus MCW220, isolated from a hot water spring. Applied Biological Research, 17(2), 139-149. http://dx.doi.org/10.5958/0974-4517.2015.00023.3

Antranikian, G., Klingeberg, M. (1991) Thermostable protease from Thermococcus sp. [Patent No. W0 1991019792.A1].

Dammak, D. F., Smaoui, S. M., Ghanmi, F., Boujelben, I., \& Maalej, S. (2016) Characterization of halo-alkaline and thermostable protease from Halorubrum ezzemoulense strain ETR14 isolated from Sfax solar saltern in Tunisia. Journal of basic microbiology, 56(4), 337-346. http://dx.doi.org/10.1002/jobm.201500475

Dutta, S., Roy, R., \& Lahiri, D. (2015)L-asparaginase and L-glutaminase from Pseudomonas aeruginosa pao1: production and some physicochemical properties. J. Microbiol. Biotech. Food Sci., 5(2), 142-146. http://dx.doi.org/10.15414/jmbfs.2015.5.2.142-146

Emi, S., Myers, D. V., \& Iacobucci, G. A. (1976). Purification and properties of the thermostable acid protease of Penicillium duponti. Biochemistry, 15(4), 842 848.http://dx.doi.org/10.1021/bi00649a018

Gupta, A., \& Khare, S. K. (2006). A protease stable in organic solvents from solvent tolerant strain of Pseudomonas aeruginosa. Bioresour. Technol., 97(15) 1788-1793.http://dx.doi.org/10.1016/j.biortech.2005.09.006

Hanzawa, S., Hoaki, T., Jannasch, H. W., Maruyama, T., (1996). An extremely thermostable serine protease from a hyperthermophilic archaeum, Desulfurococcus strain SY, isolated from a deep-sea hydrothermal vent. J. Mar Biotech., 4(2) 121-126.

Hingole, S. S., \& Pathak, A. P. (2013). Report on efficient salt stable Azospirillum a Lonar Soda Lake isolate. Sci. Res. Reporter, 3(2), 200-203.

Jadhav, S. R. \& Pathak, A. P., (2015). Report on physicochemical analysis of Unapdev hot water spring, Jalgaon, Maharashtra. Int. J. Innov. Biol. Res., 4(1), 13.http://dx.doi.org/10.1111\%2Fijibr.v4i1.91

Jadhav, S. R., \& Pathak, A. P. (2015a). Identification, characterization of an efficient thermostable multi enzyme producer from Unapdev Hot Spring. National conference on current trends in aquaculture, organized by Indira Gandhi college, Cidco, Nanded, 53, 69-71.

Jordan, J. N., Xin-Li L. (1993). Thermostable acid protease from Sulfolobus acidocaldarius [Patent No. US 5215907 A].

Joshi, A. A., Kanekar, P. P., Kelkar, A. S., Shouche, Y. S., Vani, A. A., Borgave, S. B., \& Sarnaik, S. S. (2008). Cultivable bacterial diversity of alkaline Lonar Lake, India. Microb. Ecol., 55(2), 163-172.http://dx.doi.org/10.1007/s00248-0079264-8

Khairnar, R. S., Mahabole, M. P., \& Pathak, A. P. (2012). Nanoactivato mediated modifications in thermostable amylase from Bacillus licheniformis Indian J. Biochem. Biophy. 49(6), 468-471.

Kolekar, Y. M., Pawar, S. P., Adav, S. S., Zheng, L. Q., Li, W. J., Shouche, Y. S., Dastager, S.G. \& Kodam, K. M. (2013). Alishewanella solinquinati sp. nov., isolated from soil contaminated with textile dyes. Current microbiology, 67(4), 454-459.http://dx.doi.org/10.1007/s00284-013-0385-7

Labrenz, M., Collins, M. D., Lawson, P. A., Tindall, B. J., Braker, G. \& Hirsch, P., (1998). Antarctobacter heliothermus gen. nov., sp. nov., a budding bacterium from hypersaline and heliothermal Ekho Lake. Int. J. Syst. Evol. Microbiol. 48(4) 1363-1372.http://dx.doi.org/10.1099/00207713-48-4-1363

Laemmli, U. K. (1970). Cleavage of structural proteins during the assembly of the head of bacteriophage T4. Nature, 227, 680-685.
Lindberg, A. A., Nord, C. E., \& Dahlbäck, A. (1977). Rapid identification and antibiotic sensitivity testing of bacteria isolated from clinical infections. Medical microbiology and immunology, 163(1), 13-24.

Liu, L., Ma, M., Cai, Z., Yang, X., \& Wang, W. (2010). Purification and properties of a collagenolytic protease produced by Bacillus cereus MBL13 strain. Food Tech. Biotech., 48(2), 151-160.

Lowry, O. H., Rosebrough, N. J., Farr, A. L., \& Randall, R. J. (1951). Protein measurement with the Folin phenol reagent. J. Biol. Chem. 193(1), 265-275.

Osho, M. B., Akpan, I., Adio, O. Q. (2015). Screening, optimization and characterization of extracellular lipase of Aspergillus niger ATCC 1015. Microbiol. $\quad$ Biotech. Food Sci., $\quad 5(2), \quad 172-176$ http://dx.doi.org/10.15414/jmbfs.2015.5.2.172-176

Pathak, A. P., \& Rathod, M. G. (2013). Production and Characterization of Alkaline Protease by Bacillus pasteurii: a Lonar Soda Lake Isolate. Innov. Res.Chem., 1(1), 22-26.

Pathak, A. P., \& Rathod, M. G. (2014). Exploration of Unkeshwar hot springs in Maharashtra for thermostable amylase producer. Res. \& Rev. in biosci., 8(7), 269-276.

Pathak, A. P., Rathod, M. G., \& Jadhav, S. R., (2014). Exploration of Lonar lake vegetation for alkaline protease producer. Proceeding of National conference on advanced studies in life sciences and medicinal plants, (Dept. Botany, Nagnath Arts, Comm. \& Sci. College, Aundha, India), 100-102.

Pathak, A. P., Rathod, M. G., Maske, S. V. (2014a). Isolation and identification of thermostable gelatinase producer from hot spring. Proceeding ofNational Seminar on Water Pollution and Solid Waste Management For Natural Resources Conservation (Thematics Publications, Anadur, Maharashtra, India). 1, 122-124.

Pathak, A. P., Sardar, A. G., \& Janaj, P. C. (2014b). Exploring the salted fish for salt stable amylase producing bacteria. Indian J. Mar. Sci., 43(10), 1967-1971.

Pathak, A. P., Rathod, M. G., \& Rampurkar, V. V. (2014c). An eco-friendly approach for thermostable amylase production using Bacillus firmus APP6: a hot spring isolate. Asiatic J. Biotech. Res, 4(4), 101-105.

Pathak, A. P., Lohagave, A. G., Rathod M. G. (2015). Exploration of paper industry effluent for isolation of efficient starchy material degrader to promote bioremediation. Int. J. Adv. Pharm. Biol. Chem. 4(4), 729-736.

Pathak, A. P., Lodge, N., Gavali, J., Rathod, M. G. (2015a). Isolation and characterization of cold-active protease producer from ice factory samples. Int. J. Adv. Pharm. Biol. Chem. 4(4), 751-754.

Pathak, A. P., \& Rathod, M. G. (2015). Cultivable bacterial diversity of terrestria thermal spring of Unkeshwar, India. J. Biochem. Technol., 5(4), 814-818.

Pathak, A. P. \& Rathod, M. G. (2015a). A report on thermostable alkaline protease producing bacteria from a terrestrial thermal spring, Indian J. Mar. Sci., 44 (7), 1104-1111.

Pathak, A. P., \& Rathod, M. G. (2015b). Production dynamics of thermostable lipase from thermophilic Bacillus subtilis isolated from thermal spring of Unkeshwar, Maharashtra. Proceeding ofNational conference on Life sciences major challenges, (Sanskar publications Pvt. Ltd., Latur, India). 50-53.

Pathak, A. P., \& Rathod, M. G. (2016). Assessment of diverse thermostable alkaline lipase producers from Unkeshwar hot spring of Maharashtra India.Concept. Pure Appl. Sci., 3(1), 1-9.

Pathak, A. P., \& Sardar, A. G. (2012). Isolation and characterization of carotenoid producing Haloarchaea from solar saltern of Mulund, Mumbai, India.Indian J. Nat. Prod. Resour, 3(4), 483-488.

Pathak, A. P., \& Sardar, A. G. (2014). Isolation and characterization of salt stable protease producing archaea from marine solar saltern of Mulund, Mumbai. Indian J. Mar. Sci, 43(3), 412-417

Polkade, A. V., Ramana, V. V., Joshi, A. A., Pardeshi, L., \& Shouche, Y. S. (2015). Rufibacter immobilis sp. nov., a novel strain isolated from high altitude saline Lake.Int. J. Syst. Evol. Microbiol.65 (5) 1592-1597. http://dx.doi.org/10.1099/ijs.0.000144

Rathod, M. G. \& Pathak, A. P. (2014). Wealth from Waste: Optimized alkaline protease production using agro-industrial residues by Bacillus alcalophilus LW8 and its biotechnological applications.J. Taibah Univ. Sci., 8(4) $307-$ 314.http://dx.doi.org/10.1016/j.jtusci.2014.04.002

Rathod, M. G., \& Pathak, A. P. (2014a). Isolation and identification of alkaline protease producer from selected alkaline habitat. Int. J. Innov. Biol. Res., 3(1), 16. http://dx.doi.org/10.1111\%2Fijibr.v3i1.70

Rathod, M. G., \& Pathak, A. P. (2016). Optimized production, characterization and application of alkaline proteases from taxonomically assessed microbia isolates from Lonar soda lake, India. Biocatalysis and Agricultural Biotechnology, 7, 164-173. http://dx.doi.org/10.1016/j.bcab.2016.06.002

Rathod, M. G., \& Pathak, A. P. (2016a). Data on optimized production and characterization of alkaline proteases from newly isolated alkaliphiles from Lonar soda lake, India. Data in brief, 8, 863-866 https://doi.org/10.1016/j.dib.2016.06.044

Rathod, M. G., \& Pathak, A. P. (2017). Evaluation of the enzymatic profile of microbial isolates from Lonar Soda Lake. Indian J. Mar. Sci., 46(1) 116-124.

Selim S. (2015). Thermostable Alkaline Protease Production by Bacillus Aryabhattai J4. The FASEB Journal, 29(1 Supplement), 573-574 
Sardar, A. G., \& Pathak, A. P. (2014). Exploring the microbiota of solar saltern of Mulund, Mumbai, India. Indian J. Mar. Sci., 43(4), 634-641.

Sharma, A., Pandey, A., Shouche, Y. S., Kumar, B., \& Kulkarni, G. (2009). Characterization and identification of Geobacillus spp. isolated from Soldhar hot spring site of Garhwal Himalaya, India. J. Basic Microbiol., 49(2), 187194.http://dx.doi.org/10.1002/jobm.200800194

Sharma, B., Agrawal, R., Singhania, R. R., Satlewal, A., Mathur, A., Tuli, D., \& Adsul, M. (2015). Untreated wheat straw: Potential source for diverse cellulolytic enzyme secretion by Penicillium janthinellum EMS-UV-8 mutant. Bioresour. Technol., 196, 518-524.http://dx.doi.org/10.1016/j.biortech.2015.08.012

Thebti, W., Riahi, Y., \& Belhadj, O. (2016). Purification and Characterization of a New Thermostable, Haloalkaline, Solvent Stable, and Detergent Compatible Serine Protease from Geobacillus toebii Strain LBT 77. BioMed research international, 2016.

Sneath, P. H. A., Mair, N. S., Sharpe, M. E., \& Holt, J. G. (1986). Bergey's manual of systematic bacteriology (Vol. 2). Baltimore: Williams \& Wilkins.

Ulfah, M., Helianti, I., Budiasihwahyuntari, N. \& Nurhayati, N., (2011)

Characterization of a new thermoalkalophilic xylanase-producing bacterial strain isolated from Cimanggu hot spring, West Java, Indonesia. Microbiology, 5(3), 139-143. http://dx.doi.org/10.5454/mi.5.3.7

Yildirim, V., Baltaci, M. O., Ozgencli, I., Sisecioglu, M., Adiguzel, A., \& Adiguzel, G. (2017). Purification and biochemical characterization of a novel thermostable serine alkaline protease from Aeribacillus pallidus C10: a potential additive for detergents. Journal of enzyme inhibition and medicinal chemistry,_32(1), 468-477. http://dx.doi.org/10.1080/14756366.2016.1261131 